\title{
DIABETES
}

\section{Human $\beta$-cell proliferation by promoting Wnt signalling}

A strategy involving stimulating Wnt signalling at the receptor level enhances the proliferation of $\beta$ cells in vitro whilst maintaining the function of these cells, new data reveal.

Islet transplantation for patients with type 1 diabetes mellitus typically requires 2-4 donors for one recipient, whereas use of one donor would minimize the risk of immune rejection. Proliferation of adult $\beta$ cells in vitro could hold the key to providing one donor for one recipient.

"In previous studies, we found that activation of the Wnt/GSK-3/ $\beta$-catenin pathway by pharmacologic inhibition of GSK-3 in combination with nutrient activation of mTOR, modestly enhanced human $\beta$-cell proliferation in vitro," says lead researcher Haytham Aly of the Washington University School of Medicine in St. Louis, MO, USA. "However, expansion of human islets was associated with a loss of insulin content and secretory function."

In the current study, the researchers aimed to engage canonical and noncanonical Wnt signalling at the receptor level to increase the proliferation of human $\beta$ cells in vitro, without losing the capacity of

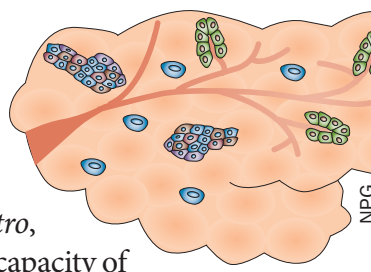
the cells to produce and secrete insulin.

The researchers treated cadaver-derived intact human islets with a conditioned medium from $L$ cells that constitutively produce Wnt-3a, R-spondin-3 and Noggin. A similar medium had previously enabled successful proliferation of mouse colonic intestinal epithelial cells. The researchers added inhibitors of ROCK and RhoA to this medium to augment cell survival.

The conditioned medium with the inhibitors lead to $\sim 20$-fold proliferation of the human $\beta$ cells above that with glucose alone. Crucially, treatment with this conditioned medium did not impair glucose-stimulated insulin secretion or decrease insulin content of the cells. In transcriptome-wide gene expression profiling and follow-up signalling studies, the researchers showed that the conditioned media treatment specifically promoted Wnt signalling.

The team now plan to determine whether the conditioned medium treatment specifically enhances the proliferation of $\beta$ cells or whether other islet cell types, in particular glucagonsecreting $\alpha$-cells, are also affected.

"This novel strategy has clear potential for use in the in vitro expansion of human islets and the subsequent treatment of impaired $\beta$-cell functional mass in type 1 diabetes mellitus and type 2 diabetes mellitus," concludes Aly.

\section{Carol Wilson}

Original article Aly, H. et al. A novel strategy to increase
the proliferative potential of adult human $\beta$-cells while
maintaining their differentiated phenotype. PLOS ONE 8,
e66131 (2013)

